\title{
HÁBITOS ORALES COMUNES: REVISIÓN DE literatura. PARTe I
}

\author{
Andrea Ocampo Parra, Res. Ortod. , Natalia Johnson García, Ortod., María Clara Lema Álvarez ${ }^{*}$, Ortod. \\ ${ }_{1}$ Universidad Cooperativa de Colombia, sede Medellín, Colombia \\ ${ }_{2}$ Universidad ces, Universidad Cooperativa de Colombia, sede Medellín, Colombia
}

Recibido: 19 de noviembre del 2012 Aprobado: 12 de febrero del 2013

* Autor de contacto: María Clara Lema Álvarez, Facultad de Odontología, Universidad Cooperativa de Colombia, 57 (4) 321 47 56, Calle 7 \# 39-197 cons. 1411 , Medellín, Colombia, correo electrónico: maria.lema@campusucc.edu.co

Cómo citar este artículo: Ocampo Parra A, Johnson García N, Lema Álvarez MC. Hábitos orales comunes: revisión de literatura. Parte I. Rev. Nac. Odontol. 2013 diciembre; 9 (edición especial): 83-90.

Resumen. Existen hábitos orales nocivos que pueden influir en el desarrollo de las maloclusiones; aunque estos no son un riesgo para la vida, sus consecuencias son un problema de salud pública. En esta revisión de literatura se propone establecer los hábitos más evaluados por los odontólogos, id entificar sus problemas y dar una orientación practica. Se realizó una búsqueda de la literatura acerca de los hábitos orales al momento de la consulta odontológica con el fin de encaminar a un rápido tratamiento para que el daño causado sea menor

Palabras clave: chupetes, hábitos, hábitos de lengua, maloclusión, onicofagia, respiración oral, succión digital.

\section{COMmon Oral Habits: A REVIEW OF THE LITERATURE. PART I}

Abstract. There are harmful oral habits that may influence the development of malocclusions; although they are not life-threatening, their consequences constitute a public health problem. This literature review seeks to establish habits more commonly evaluated by dentists, identify their problems and provide practical guidance. A search was made of the literature on oral habits to find rapid treatment with the aim of limiting the damage.

Keywords: pacifiers, habits, tongue habits, malocclusion, nail biting, mouth breathing, finger sucking. 


\section{Introducción}

Por definición, un hábito es la facilidad que adquiere una persona para repetir una determinada actividad [1]. Los hábitos orales son comportamientos parafuncionales que pueden ser normales en determinado momento de la vida y se ha reportado que su persistencia en el tiempo puede conducir al desarrollo de una maloclusión [2]. Existen corrientes acerca del origen de la maloclusión que subrayan la capacidad del medio ambiente y de los hábitos anormales para alterar los tejidos blandos y a su vez influir en el desarrollo y crecimiento craneofacial [3]. De esta forma, cuando la actividad excede la tolerancia fisiológica individual, se presenta una alteración y puede ocurrir un daño a nivel dental, muscular o articular [4].

Un enfoque de tratamiento exitoso debe estar encaminado a modificar los patrones funcionales de los tejidos orales [1]. Suspender un hábito no funcional requiere la cooperación del paciente y su madurez para entender las consecuencias de un hábito persistente $[5,6]$.

El propósito del presente artículo es revisar la literatura actual sobre los hábitos orales e identificar los más frecuentes, focalizándose en su incidencia, etiología y diversos enfoques de tratamiento.

\section{Hábitos orales}

En su estudio, Subtelny hace referencia al primer reporte en la literatura referente a los hábitos realizado por Olson en 1929, el cual hace mención a diferentes tipos de hábitos, como aquellos que comprometen los dedos (comerse las uñas, rascarse, frotar los ojos, hurgar la nariz, halar las orejas, halar el cabello entre otros) [1]. En 1995, Josell [7] propuso una nueva clasificación de los hábitos orales en 3 grupos: (a) de tipo nervioso, como mordisqueo de labio o carrillo, de objetos, empuje lingual, deglución infantil, onicofagia, aplicar presión en los dientes usando el dedo o un objeto; (b) hábitos profesionales: sostener objetos en la boca, como instrumentos musicales; (c) hábitos ocasionales: cigarrillo o pipa, masticación de tabaco, cepillado inadecuado, succión digital o de labio [8].

\section{Incidencia}

De acuerdo con Olson (1929) [1], el hábito más común es succión digital, seguido de onicofagia, morder el labio y empuje lingual; según Josell (1995) [7], el más frecuente es onicofagia y morder objetos. Gavish (2000) [4] afirma que son más frecuentes el masticar chicle, masticar hielo y morder objetos. En el 2002, Bosnjak [8] identificó que el hábito más frecuente fue morder objetos u onicofagia $(28,07 \%)$, succión digital $(18,42 \%)$, empuje lingual $(17,25 \%)$ y morderse los labios o carrillos $(16,96 \%)$. Un estudio realizado en México en el 2009 encontró una incidencia de un 35\% en el hábito de onicofagia, siendo este el hábito más frecuente, seguido por la succión digital $(8,5 \%)$ y succión labial (8\%) [9]. La búsqueda de literatura realizada para la presente revisión no arrojó ninguna cita acerca de hábitos en población colombiana.

\section{Diagnóstico}

Para realizar un diagnóstico adecuado es necesario un buen examen clínico, el cual debe incluir la evaluación de la función y de la morfología de la lengua, los labios y la musculatura orofacial [10]. También se deben analizar los dientes individualmente y el tipo de oclusión. Es importante conocer el patrón funcional normal de las estructuras orales para poder distinguir los patrones anormales [1].

\section{Función normal}

\section{Deglución}

Es una acción neuromuscular compleja (automática), que se logra por medio de fuerzas y movimientos sincronizados ocurridos dentro de la boca, la faringe, la laringe y el esófago, con interrupciones de la respiración, cuya finalidad es llevar el bolo alimenticio a la faringe [11].

Muchos profesionales - como odontólogos y fonoaudiólogos- han aceptado una descripción de deglución normal en la cual los músculos de la expresión facial no se usan, los músculos de la masticación unen los maxilares y la lengua permanece confinada dentro de los arcos dentales, esta realiza un movimiento ondulatorio de adelante hacia atrás para transportar el bolo alimenticio [1].

Las variaciones en la deglución se presentan especialmente en la región anterior, independientemente de la configuración dentoesquelética que un individuo pueda tener. La lengua se adapta al patrón oclusal existente, al tratar de buscar el selle $[12,13]$. Un ejemplo de esto es común en la protrusión maxilar, en la que hay 
un overjet aumentado y la lengua se protruye más allá de los incisivos inferiores en un intento de contactar las superficies linguales de los incisivos superiores durante la deglución [14]. Sin embargo, autores como Ardran y Kemp en 1955 [13], y Rogers en 1961 [14], encontraron patrones anormales de deglución en pacientes con oclusión normal.

\section{Deglución infantil}

Se caracteriza por posicionar la lengua del niño debajo de pezón contactando el labio inferior y deglutiendo con los labios juntos y los maxilares separados [7, 8]. Este patrón es normal durante los primeros doce meses de vida, cuando la lengua se interpone entre los rebordes que se encuentran en mordida abierta. Si luego de este tiempo dicho patrón se hace persistente, se considera como hábito de deglución infantil [15].

Muchos autores mencionan la deglución con empuje lingual como deglución infantil persistente, pero una verdadera deglución de tipo infantil persistente es escasa. Los niños con algún tipo de compromiso neurológico mantienen la deglución infantil [8].

\section{Hábitos orales}

\section{Empuje lingual}

Es la protrusión de la lengua entre los incisivos o los segmentos bucales durante el acto de la deglución [16].

En 1961, Fletcher, Casteel y Bradley [17] mostraron una incidencia proporcional de empuje lingual que disminuye con la edad. Su estudio reportó que más de la mitad de los pacientes entre 6 y 7 años de edad tenían empuje lingual, y menos del $25 \%$ de los pacientes entre 16 y 18 años mostraban empuje lingual.

El empuje lingual se puede clasificar como simple o complejo [18]; en el simple existe una contracción de labios, músculos mentonianos y elevadores mandibulares; los dientes posteriores están en oclusión, la lengua se encuentra en protrusión pero existe un engranaje cuspídeo preciso y seguro. En el empuje lingual complejo existe un empuje lingual y deglución con dientes separados, contracción en labios, músculos faciales y mentonianos, no hay contracción elevadores mandibulares y hay pobre adaptación e inestabilidad en engranaje cuspídeo.

En 1965 fue propuesta por Brauer [15] una clasificación alterna de la deglución con empuje lingual con base en la deformidad producida y no en la etiología. Esta se divide en cuatro grupos:

- Tipo I: empuje lingual no deformante.

- Tipo II: empuje lingual deformante anterior (subgrupo 1: mordida abierta anterior, subgrupo 2: asociado protrusión de dientes anteriores, subgrupo 3: mordida cruzada posterior).

- Tipo III: empuje lingual deformante lateral (subgrupo 1: mordida abierta posterior, subgrupo 2: mordida cruzada posterior, subgrupo 3: sobremordida profunda).

- Tipo IV: empuje lingual deformante anterior y lateral (subgrupo 1: mordida abierta anterior y posterior, subgrupo 2: asociado con protrusión de dientes anteriores, subgrupo 3: asociado con mordida cruzada posterior).

Para tratar el empuje lingual es importante modificar los patrones funcionales de los tejidos orales [1] utilizando la ayuda del fonoaudiólogo - para una terapia funcional-y del ortodoncista o cirujano para un tratamiento ortodóncico o un reposicionamiento quirúrgico de los maxilares [19]. No se debe tratar en pacientes que presenten discrepancias esqueléticas considerables que no se vayan a intervenir quirúrgicamente, aquellos con daño neurológico grave o tamaño de la lengua anormal, ya que no hay control de la función de la musculatura orofacial [1].

\section{Hábito de succión digital y de pulgar}

Existen esencialmente dos formas de succión: la nutritiva y la no nutritiva, ambas provocan sensación de calidez y seguridad; a esta última correspondería el hábito del chupete. La succión no nutritiva de dedos, chupetes y otros elementos no relacionados con la ingesta de nutrientes se considera una actividad normal en el desarrollo fetal y neonatal hasta los 18 meses de vida, edad a partir de la cual pueden presentarse consecuencias nocivas [20].

En este hábito se posiciona el pulgar u otros dedos dentro de la boca muchas veces durante el día y la noche, ejerciendo una presión definida al succionar [2]. Ha sido asociado con un desbalance psicológico que constituye una forma errónea de solucionar problemas, y está relacionado con estados de ansiedad e inestabilidad emocional [21]. Existen 4 categorías descritas por Subtelny [1] para posicionar el dedo. Estas son: 
1. El pulgar entra en la boca considerablemente más allá de la primera articulación o nudillo. Este va a ocupar una gran porción de la bóveda del paladar duro presionado contra la mucosa palatina y el tejido alveolar. El incisivo inferior es presionado hacia el pulgar, o lo contacta.

2. El pulgar no va directamente al área de la bóveda del paladar duro, sin embargo con frecuencia entra en la boca alrededor de la primera articulación o anterior a esta. Puede observarse contacto entre incisivos inferiores y el pulgar.

3. El pulgar pasa completamente a la cavidad oral y se aproxima a la bóveda del paladar duro como en el primer grupo, aunque este grupo varía de los otros en que el incisivo inferior no contacta el pulgar en ningún momento durante el proceso de succión.

4. El pulgar no progresa apreciablemente dentro de la boca. El incisivo inferior hace contacto a nivel de la uña.

Durante la dentición primaria los hábitos de succión no producen efectos nocivos; sin embargo, cuando un niño adquiere un hábito de succión no nutritivo prolongado, este con frecuencia conduce a maloclusión [2]. Si el hábito de succión de pulgar o de otro dedo persiste más allá del tiempo en que los dientes permanentes comienzan a erupcionar, se desarrolla una maloclusión [22].

El pulgar es el factor causante primario de la maloclusión anterior y no la actividad protrusiva de la lengua. En respuesta análoga a la pregunta de qué vino primero si la gallina o el huevo, las observaciones indican que la succión digital es primero, y luego se presenta la actividad protrusiva de la lengua [1].

El desarrollo de una mordida abierta anterior se relaciona con la duración más que con la intensidad del hábito. Es probable desarrollar maloclusiones luego de 18 meses de presentar el hábito [2].

La maloclusión resultante se caracteriza por incisivos superiores espaciados y proinclinados, inferiores retroinclinados, mordida abierta anterior y un arco maxilar estrecho, y dependiendo de cómo se chupe el dedo, los incisivos inferiores también se pueden vestibularizar $[2,6]$.

Para realizar el tratamiento es importante tener en cuenta la edad del paciente; es necesario que sea lo suficientemente maduro para entender las consecuencias de un hábito persistente. La terapia no debe ser un castigo sino una ayuda, y es de vital importancia contar con la cooperación de los padres para un tratamiento exitoso [6]. Se puede iniciar con terapias simples como recordatorios, bandas adhesivas en los dedos o guantes en ambas manos a la hora de dormir [5]. Si dicha terapia no es efectiva, se puede iniciar con aparatología fija como la rejilla larga o un Quad Hélix con rejilla para pacientes que también tengan mordidas cruzadas por dicho habito [6].

También existen terapias con brackets de hábito o "spurs" que se cementan directamente en la superficie palatina de incisivos superiores y van a desencadenar un reflejo nociceptivo (dolor). Estos ejercen un control continuo por medio de un mecanismo de biorretroalimentación que "informa" al paciente acerca de la mala posición de la lengua [23].

\section{Uso de chupetes}

La utilización del chupete es motivo de controversia entre los profesionales de la salud, que recomiendan o desaconsejan su uso basándose algunas veces en experiencias personales y no siempre en pruebas científicas [24].

El uso de chupetes obedece a la necesidad de succión, la cual forma parte del funcionamiento saludable y esencial para el desarrollo de la mandíbula y la cavidad oral de acuerdo con la edad del niño. De esta forma, los chupetes son un apoyo importante durante este intervalo del desarrollo [25]. Esta necesidad fisiológica de succión es más importante durante los 6 primeros meses de vida; después de esa edad, el uso del chupete tiende a ser un hábito que brinda sensación de seguridad [26].

De acuerdo con el rango de edad, el uso del chupete es más frecuente a la edad de tres años [9], resultado que concuerda con los hallazgos de Cepero [27]. Aliboni [26] encontró que los primogénitos usan menos chupete que aquellos que tienen hermanos. Asimismo, los padres que usaron chupete durante su infancia por tiempo prolongado, extienden su uso en sus hijos [26]. Además, los niños que no usan el chupete pueden presentar otro hábito de succión: 33\% dedo pulgar, 27\% biberón, 13\% una sábana o "trapito", 3,7\% otros elementos y $22,2 \%$ nada.

Al llevar a posición el chupete, la lengua es forzada hacia una posición inferior en la parte anterior de la boca, ejerciendo una presión lateral incrementada sobre los caninos y primeros molares inferiores. La falta de soporte palatino de la lengua resultará en un arco superior más estrecho, y la presión de la lengua ampliará el arco inferior. Estos cambios crean una desarmonía 
transversal que incrementa la tendencia a desarrollar una mordida cruzada posterior [28].

El uso del chupete aumenta 6 veces más la probabilidad de desarrollar maloclusiones; en pacientes que lo usan, se encontró un incremento en la maloclusión únicamente cuando estos dejan de usar el chupete después de los 2 años de edad, con una probabilidad de maloclusión 13,6 veces mayor [15]. Se ha estimado que debe estar presente por lo menos 2 años para que tenga efecto en el maxilar [29], reduciendo la distancia intercanina $[28,30]$, y 3 años para aumentar el ancho intercanino mandibular [29].

Mucho se ha discutido sobre los perjuicios y beneficios de la utilización del chupete, tales como mordida abierta, mordida cruzada posterior, profundidad del paladar aumentada [31], incremento en la incidencia de otitis media, candidiasis oral [26], problemas dentales, acortamiento del tiempo de amamantamiento, dificultades en la lactancia, riesgo de accidentes, hipersensibilidad al látex y ulceras orales [24]. Algunos beneficios incluyen disminución del riesgo de muerte súbita del lactante, efecto analgésico y estímulo de la succión no nutritiva en niños pretérmino y a término [24].

Es importante conocer los tipos de chupos que se encuentran disponibles en el mercado, y es esencial usar diferentes tamaños de chupetes para poder ir a la par con el rápido crecimiento de la cavidad bucal y la mandíbula, especialmente en el primer año de vida [29]. No todos los chupete son adecuados, dado que su forma puede afectar el desarrollo de la mandíbula y la cavidad bucal. Existen chupetes planos, redondos y ortodóncicos [25].

El chupete redondo puede inhibir el desarrollo anatómico y fisiológico de la boca, o puede empujar la lengua hacia una dirección no deseada. El uso de este se ha asociado con distancias intercaninas e intermolares disminuidas en maxilar superior y una incompetencia labial [15]. El chupete plano ofrece un movimiento fisiológico de la lengua que puede promover el cierre completo de los labios [29].

Alertados por las altas tasas de anomalías mandibulares en niños, los doctores W. Balters y A. Müller desarrollaron el chupón ortodóncico con una forma adaptada a la mandíbula, indicado para un desarrollo adecuado mandibular. Es una medida profiláctica y de apoyo para el desarrollo fisiológico de la mandíbula y la cavidad bucal [29].

Los chupetes para niños de 0 a 4 meses de edad tienen una forma que se asemeja a la del pezón de la madre, factor que hace que el bebé sienta cierta satisfacción emocional cuando está con este chupete. Para la edad de 4 a 5 meses los chupetes son diseñados con una base delgada que sirve para que los bebés puedan cerrar los labios de manera natural y estimular la respiración nasal. Para niños con más de 8 meses la base del chupete es delgada, pero tiene una superficie irregular que estimula el movimiento de la lengua, colaborando en el desarrollo del lenguaje [29].

La Academia Americana de Pediatría recomienda retirar el chupete cuando el niño ya tiene un año, para evitar otros efectos adversos derivados de su utilización. Asimismo, ante la evidencia actual que sugiere una disminución del riesgo del síndrome de muerte súbita del lactante, la Sociedad Pediátrica Canadiense señala que los chupetes no deben ser desaconsejados, e indica que su uso precoz debe alertar a los profesionales sobre dificultades en la lactancia, y recomiendan su empleo en las unidades de cuidados intensivos neonatales para la succión no nutritiva y el confort de los pretérminos y lactantes enfermos [24].

En conclusión, se recomienda en niños lactantes no iniciar el uso del chupete antes de los 15 días de vida, restringirlo a los 8 meses y suprimirlo al año, lo cual no aplica para niños pretérmino o cuando se presentan dificultades en la lactancia [20]. Corresponde a los profesionales de la salud proporcionar a los padres una información equilibrada, no sesgada, sobre la evidencia disponible de los beneficios y perjuicios del uso del chupete, que les ayude a tomar sus decisiones. Los responsables son, en último término, los padres [24].

\section{Onicofagia}

La onicofagia hace referencia al hábito de morderse las uñas. Este es un hábito común y se observa tanto en niños como en adultos; se caracteriza por lesiones repetidas, es de tipo autodestructivo y agresivo [32]. Se conoce como un hábito de transferencia de la succión del pulgar, ya que este tiende a ser abandonado durante el tercer año de vida. La necesidad de morder y comer incluso las uñas se vincula con un estado psicoemocional de ansiedad [5]. Se observa en edades entre los 4 y 6 años; se estabiliza entre los 7 y 10 años; aumenta considerablemente durante la adolescencia; la incidencia se reduce cuando aumenta la edad [5, 33]. La causa básica de la onicofagia es difícil de determinar, puede relacionarse con ansiedad, imitación y estrés [34]. Los pacientes bajo tratamiento ortodóncico que sufren este hábito pueden desencadenar reabsorción radicular por la aplicación de fuerzas no fisiológicas extras. A nivel 
oral también puede encontrarse apiñamiento, rotación, desgaste, fracturas, protrusión de incisivos superiores, destrucción periodontal de la zona anterior, problemas estomacales, onicomicosis y paroniquia [35].

Para dejar el hábito, el paciente debe ser motivado y ser consciente de la necesidad de abandonarlo; aquí el rol profesional adquiere relevancia, ya que ofrece sugerencias útiles para la superación de la adicción [36]. Castigar, ridicularizar y amenazar no es útil, pues esto a menudo agrava el problema o lo reemplaza con trastornos psicológicos graves, y podría provocar conflictos sociales y sentimientos de culpa [37].

Para los casos leves de onicofagia no se necesita tratamiento, mientras que para situaciones más graves el tratamiento debe involucrar la eliminación de los factores emocionales que induzcan el hábito. En algunos casos, un poco más de atención y la comprensión son suficientes. Se recomienda actividades al aire libre o actividades con las manos [5]. Un enfoque multidisciplinario debe centrarse en los esfuerzos para contribuir a la autoconfianza y la autoestima del paciente $[5,38]$.

\section{Interposición labial}

El hábito de succión del labio inferior puede manifestarse en cualquier edad. Con frecuencia se acompaña de grietas, sequedad, erosión, irritación de uno o de ambos labios o borde bermellón [6, 39]. Por lo general se asocia con una protrusión labial de los incisivos superiores, y un desplazamiento lingual de los incisivos inferiores. Puede haber retracción o dehiscencia de la encía de los incisivos inferiores [6, 39]. Este hábito puede ser la consecuencia más que la causa de una maloclusión clase II división 1, y clínicamente se observa el surco mentolabial pronunciado [40].

El hábito de interposición labial puede contribuir a un desequilibrio muscular orofacial asociado con alteraciones en el crecimiento óseo, malposición dental y deformaciones dentofaciales. La manifestación de una maloclusión adquirida varía según el tipo, la localización, severidad, frecuencia y longevidad de la costumbre, pero la eliminación del hábito es fundamental para el tratamiento y la estabilidad futura [39].

Para este hábito se puede utilizar la bompereta labial, la cual inhibe la presión del músculo orbicular de los labios y del músculo mentalis, este recuperador de espacio vestibulariza incisivos, aumenta longitud y ancho del arco, y alivia el apiñamiento en el arco inferior. Los resultados dependen de la posición del labio contra la bompereta, la altura del escudo labial y la duración de uso $[6,39,41,42]$.

\section{Respiración oral}

La influencia del modo de respiración en el crecimiento y desarrollo dentofacial ha sido objeto de controversia. De acuerdo con la teoría funcional de Moss, la respiración nasal permite un adecuado crecimiento y desarrollo del complejo craneofacial y dentofacial [43]. Los niños con respiración oral tienen 10 veces más probabilidad de desarrollar una maloclusión en comparación con quienes respiran por la nariz [44]; estos pacientes se muestran aperezados en las mañanas y pueden presentar un deficiente rendimiento escolar [45].

Su etiología es multifactorial, puede ir desde una obstrucción anatómica (hipertrofia de amígdalas palatinas, desviación del septum nasal, pólipos, hipertrofia de cornetes) hasta traumas nasales y rinitis alérgica [46].

La causa más común de la respiración oral en la población pediátrica es la obstrucción nasal, específicamente la hipertrofia adenoidea. En los niños este fenómeno es importante debido a su influencia adversa en el crecimiento y desarrollo [44, 47]. La respiración oral puede conducir a una fuerza labial de cierre debilitada, probablemente porque se mantienen los labios separados rutinariamente y no se ejercitan efectivamente los músculos periorales [44].

La obstrucción nasal crónica conduce a respiración oral; posición anterior o inferior de la lengua; mordida abierta anterior; labios medio abiertos; posición baja de la mandíbula; rotación horaria; elongación de la altura facial inferior; tonicidad reducida de la musculatura orofacial; desarmonía en el crecimiento y desarrollo de las estructuras orofaciales; maxilar estrecho; subdesarrollo de la mandíbula; alteraciones en la posición de la cabeza y protrusión de incisivos superiores [44, 47].

El tratamiento del respirador oral requiere un enfoque multidisciplinario: puede requerir tratamiento quirúrgico si la causa es obstrucción nasal (amígdalas, adenoides, pólipos, etcétera); interconsulta con otorrino para tratar problemas de asma, rinitis alérgica, bronquitis, entre otros; interconsulta con fonoaudiólogo para enseñar a respirar correctamente y tratar los problemas del habla; tratamiento ortodóncico con aparatos de disyunción, corrigiendo la alteración transversal asociada con este hábito por medio de expansión rápida maxilar, terapia que ha demostrado ser efectiva en la morfología del arco dental superior, mejorando 
las dimensiones de la cavidad nasal y en la resistencia de las vías aéreas hasta en un $45 \%$. En el largo plazo, los pacientes reportan una mejoría en la respiración nasal $[45,48-50]$.

\section{Conclusiones}

Luego de la revisión de literatura se puede concluir que los hábitos orales nocivos llevan a maloclusiones y estas dependerán de la frecuencia, fuerza y duración de los hábitos. En la consulta, el odontólogo tiene la responsabilidad de identificar e intervenir oportunamente los hábitos parafuncionales, especialmente en población infantil, para evitar o interceptar alguna maloclusión.

\section{Referencias}

[1] Subtelny JD. Oral habits. Studies in form, function, and therapy. Angle Orthod. 1973; 43: 349-83.

[2] Singh SP, Utreja A, Chawla HS. Distribution of malocclusion types among thumb suckers seeking orthodontic treatment. J Indian Soc Pedod Prev Dent. 2008; 26(Suppl 3): S114-7.

[3] Aguilar M. Relación entre hábitos nocivos y maloclusiones en una muestra de 525 pacientes de ortodoncia. Revista Latinoamericana de Ortodoncia y odontopediatria. 2011; http://www.ortodoncia.ws/publicaciones/2011/art25.asp

[4] Gavish A, Halachmi M, Winocur E, Gazit E. Oral habits and their association with signs and symptoms of temporomandibular disorders in adolescent girls. J Oral Rehabil. 2000; 27: 22-32.

[5] Tanaka OM, Vitral RW, Tanaka GY, Guerrero AP, Camargo ES. Nailbiting, or onychophagia: a special habit. Am J Orthod Dentofacial Orthop. 2008; 134: 305-8.

[6] Cárdenas D. Fundamentos de odontología pediátrica. 2a. ed. Bogotá: CIB; 2000.

[7] Josell SD. Habits affecting dental and maxillofacial growth and development. Dent Clin North Am. 1995; 39: 851-60.

[8] Bosnjak A, Vucicevic-Boras V, Miletic I, Bozic D, Vukelja M. Incidence of oral habits in children with mixed dentition. J Oral Rehabil. 2002; 29: 902-5.

[9] Murrieta F. Prevalence of non-nutrituve habits in a group of preschool children in NNezahualcoyotl, Mexico. Bol Med Hosp Infant. Mex. 2011; 24-30.

[10] Barberia E, Lucavechi T, Cardenas D, Maroto M. An atypical lingual lesion resulting from the unhealthy ha- bit of sucking the lower lip: clinical case study. J Clin Pediatr Dent. 2006; 30: 280-2.

[11] Okeson J. Tratamiento de oclusión y afecciones temporomandibulares. 6a . ed. Madrid: Mosby; 2003.

[12] Straub WJ. The etiology of the perverted swallowing habit. Am J Orthod. 1951; 37: 603-10.

[13] Ardran GM, Kemp FH, Truelove SC. A radiological study of the movements of the tongue, pharynx and oesophagus in acrosclerosis. Gastroenterología. 1953; 79: 361-74.

[14] Rogers J. Swallowing patterns of a normal population sample compared to those of patients from an orthodontic practice. Am J Orthod. 1961; 47: 674-89.

[15] Gois EG, Ribeiro-Junior HC, Vale MP, Paiva SM, SerraNegra JM, Ramos-Jorge ML et al. Influence of nonnutritive sucking habits, breathing pattern and adenoid size on the development of malocclusion. Angle Orthod. 2008; 78: 647-54.

[16] Brauer JS, Holt TV. Tongue Thrust Classification. Angle Orthod. 1965; 35: 106-12.

[17] Fletcher SG, Casteel RL, Bradley DP. Tongue-thrust swallow, speech articulation, and age. J Speech Hear Disord. 1961; 26: 201-8.

[18] Cleall JF. Deglutition: A Study of Form and Function. Am J Orthod. 1965; 51: 566-94.

[19] Subtelny JD. Malocclusions, orthodontic corrections and orofacial muscle adaptation. Angle Orthod. 1970; 40: 170-201.

[20] Matínez L. Uso del chupete: beneficios y riesgos. Anales Españoles de Pediatría. 2000; 53: 6.

[21] Sweet CA. Thumb- and finger-sucking by children. Am J Orthod. 1948; 34: 1017.

[22] Zadik D, Stern N, Litner M. Thumb- and pacifier-sucking habits. Am J Orthod. 1977; 71: 197-201.

[23] Meyer-Marcotty P, Hartmann J, Stellzig-Eisenhauer A. Dentoalveolar open bite treatment with spur appliances. J Orofac Orthop. 2007; 68: 510-21.

[24] Lozano M. Uso del chupete y lactancia materna. An Pediatric (Barc). 2011; 271e: 1-5.

[25] Aznar T, Galan AF, Marin I, Dominguez A. Dental arch diameters and relationships to oral habits. Angle Orthod. 2006; 76: 441-5.

[26] Aliboni V. Uso del chupete: hallazgos preliminares. Arch Argent Pediatr. 2002; 2: 114-119.

[27] Cepero S. Intervención educativa en escolares de 5 y 6 años con hábitos bucales deformantes. Rev Cubana Estomatol. 2007; versión on-line.

[28] Larsson E. Sucking, chewing, and feeding habits and the development of crossbite: a longitudinal study of girls 
from birth to 3 years of age. Angle Orthod. 2001; 71: 116-9.

[29] Heb C. Los chupones satifacen la necesidad natural de succionar. Taller de expertos: "efectos de los chupones en la mandíbula y desarrollo bucal”. Hamburgo. 2010. http://www.nukargentina.com.ar/userdata/Soothers_ ES.pdf

[30] Ogaard B, Larsson E, Lindsten R. The effect of sucking habits, cohort, sex, intercanine arch widths, and breast or bottle feeding on posterior crossbite in Norwegian and Swedish 3-year-old children. Am J Orthod Dentofacial Orthop. 1994; 106: 161-6.

[31] Warren JJ, Bishara SE, Steinbock KL, Yonezu T, Nowak AJ. Effects of oral habits' duration on dental characteristics in the primary dentition. J Am Dent Assoc. 2001; 132: 1685-93; quiz 1726.

[32] Pelc AW, Jaworek AK. Interdisciplinary approach to onychophagia. Przegl Lek. 2003; 60: 737-9.

[33] Ballinger BR. The prevalence of nail-biting in normal and abnormal populations. Br J Psychiatry. 1970; 117: 445-6.

[34] Hill JM. Nail biting; incidence, allied personality traits and military significance. Am J Psychiatry. 1946; 103: 185-7.

[35] Odenrick L, Brattstrom V. The effect of nailbiting on root resorption during orthodontic treatment. Eur J Orthod. 1983; 5: 185-8.

[36] Leung AK, Robson WL. Nailbiting. Clin Pediatr (Phila). 1990; 29: 690-2.

[37] Malone AJ, Massler M. Index of nailbiting in children. J Abnorm Psychol. 1952; 47: 193-202.

[38] Schneider PE, Peterson J. Oral habits: considerations in management. Pediatr Clin North Am. 1982; 29: 523-46.

[39] Germec D, Taner TU. Lower lip sucking habit treated with a lip bumper appliance. Angle Orthod. 2005; 75: 1071-76.
[40] Bjerregaard J, Bundgaard AM, Melsen B. The effect of the mandibular lip bumper and maxillary bite plate on tooth movement, occlusion and space conditions in the lower dental arch. Eur J Orthod. 1980; 2: 257-65.

[41] Davidovitch M, McInnis D, Lindauer SJ. The effects of lip bumper therapy in the mixed dentition. Am J Orthod Dentofacial Orthop. 1997; 111: 52-8.

[42] Ashish DI, Mostafa YA. Effect of lip bumpers on mandibular arch dimensions. Am J Orthod Dentofacial Orthop. 2009; 135: 106-9.

[43] Moss ML, Salentijn L. The primary role of functional matrices in facial growth. Am J Orthod. 1969; 55: 56677 .

[44] Harari D, Redlich M, Miri S, Hamud T, Gross M. The effect of mouth breathing versus nasal breathing on dentofacial and craniofacial development in orthodontic patients. Laryngoscope. 2010; 120: 2089-93.

[45] Goncalves R de C, Raveli DB, Pinto Ados S. Effects of age and gender on upper airway, lower airway and upper lip growth. Braz Oral Res. 2011; 25: 241-7.

[46] Warren DW. Effect of airway obstruction upon facial growth. Otolaryngol Clin North Am. 1990; 23: 699-712.

[47] Ngan P, Fields HW. Open bite: a review of etiology and management. Pediatr Dent. 1997; 19: 91-8.

[48] Bishara SE, Staley RN. Maxillary expansion: clinical implications. Am J Orthod Dentofacial Orthop. 1987; 91: 3-14.

[49] Hershey HG, Stewart BL, Warren DW. Changes in nasal airway resistance associated with rapid maxillary expansion. Am J Orthod. 1976; 69: 274-84.

[50] Oliveira de Felippe NL, Da Silveira AC, Viana G, Kusnoto B, Smith B, Evans CA. Relationship between rapid maxillary expansion and nasal cavity size and airway resistance: short- and long-term effects. Am J Orthod Dentofacial Orthop. 2008; 134: 370-82. 